\title{
UNDERSTANDING THE ROLE OF DEFECT PRODUCTION IN RADIATION EMBRITTLEMENT OF REACTOR PRESSURE VESSELS*
}

\author{
D. E. Alexander and L. E. Rehn \\ Materials Science Division \\ 9700 S. Cass Avenue \\ Argonne National Laboratory \\ Argonne, IL 60439
}

G. R. Odette, G. E. Lucas, D. Klingensmith, and D. Gragg

Dept. of Mechanical and Environmental Engr.

University of California

Santa Barbara, CA 93106

July 1999

The submitted manuscript has been created
by the University of Chicago as Operator of
Argonne National Laboratory ("Argonne")
under Contract No. W-3i-109-ENG-38 with
the U.S. Department of Energy. The U.S.
Government retains for itself, and others
acting on its behalf, a paid-up, non
exclusive, irrevocable worldwide license in
said article to reproduce, prepare derivative
works, distribute copies to the public, and
perform publicly and display publicly, by or
on behalf of the Government.

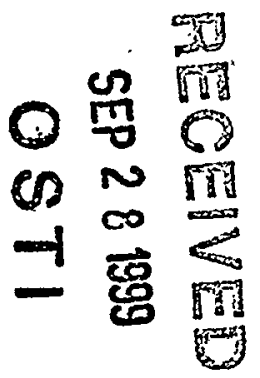

To be submitted to the $9^{\text {th }}$ International Conference on Environmental Degradation of Materials in Nuclear Power Systems-Water Reactors, Newport Beach, CA, August 1-5, 1999. *Work supported by the U.S. Department of Energy, Basic Energy Sciences-Materials Sciences,
under contract \#W-31-109-ENG-38. 


\section{DISCLAIMER}

This report was prepared as an account of work sponsored by an agency of the United States Government. Neither the United States Government nor any agency thereof, nor any of their employees, make any warranty, express or implied, or assumes any legal liability or responsibility for the accuracy, completeness, or usefulness of any information, apparatus, product, or process disclosed, or represents that its use would not infringe privately owned rights. Reference herein to any specific commercial product, process, or service by trade name, trademark, manufacturer, or otherwise does not necessarily constitute or imply its endorsement, recommendation, or favoring by the United States Government or any agency thereof. The views and opinions of authors expressed herein do not necessarily state or reflect those of the United States Government or any agency thereof. 


\section{DISCLAIMER}

Portions of this document may be illegible in electronic image products. Images are produced from the best available original document. 


\title{
UNDERSTANDING THE ROLE OF DEFECT PRODUCTION IN RADIATION EMBRITTLEMENT OF REACTOR PRESSURE VESSELS
}

\author{
Dale E. Alexander and L.E. Rehn \\ Materials Science Division \\ Argonne National Laboratory \\ Argonne, IL, 60439 \\ G.R. Odette, G.E. Lucas, D. Klingensmith and D. Gragg \\ Department of Mechanical and Environmental Engineering \\ University of California-Santa Barbara \\ Santa Barbara, CA, 93106.
}

\begin{abstract}
Comparative experiments using high energy (10 MeV) electrons and test reactor neutrons have been undertaken to understand the role that primary damage state has on hardening (embrittlement) induced by irradiation at $300^{\circ} \mathrm{C}$. Electrons produce displacement damage primarily by low energy atomic recoils, while fast neutrons produce displacements from considerably higher energy recoils. Comparison of changes resulting from neutron irradiation, in which nascent point defect clusters can form in dense cascades, with electron irradiation, where cascade formation is minimized, can provide insight into the role that the in-cascade point defect clusters have on the mechanisms of embrittlement. Tensile property changes induced by $10 \mathrm{MeV}$ electrons or test reactor neutron irradiations of unalloyed iron and an $\mathrm{Fe}-0.9 \mathrm{wt} . \% \mathrm{Cu}-1.0 \mathrm{wt} . \% \mathrm{Mn}$ alloy were examined in the damage range of $9.0 \times 10^{-5} \mathrm{dpa}$ to $1.5 \times 10^{-2} \mathrm{dpa}$. The results to date showed the ternary alloy experienced substantially greater embrittlement in both the electron and neutron irradiated samples relative to unalloyed iron. Surprisingly, despite their disparate nature of defect production, similar embrittlement trends with increasing radiation damage were observed for electrons and neutrons in both the ternary and unalloyed iron.
\end{abstract}




\section{Introduction}

Understanding microstructural evolution in nuclear reactor pressure vessels (RPVs) is critical for predicting and modeling radiation embrittlement. It is however a complex process involving a variety of environmental variables (irradiation flux, fluence, temperature, etc.) and metallurgical variables (alloy composition, preexisting microstructure, etc.) [1]. Rate theory approaches have been used to incorporate these various variables and model the evolution of microstructural features such as alloy phases [e.g. copper-rich precipitates (CRPs)] and defect clusters [1-4] with fluence. Embrittlement is caused by yield stress increases introduced by these hardening centers (hence the terms "embrittlement" and "hardening" are used interchangeably in the remainder of the paper). As available computing power increases, atomistic simulations of microstructural evolution such as molecular dynamics (MD) and kinetic Monte Carlo have become more practical [5-10]. While offering the potential to eventually supplant mean-field rate theory, these approaches are in their early application stages and are subject to current computational and physical limitations (e.g. inadequate interatomic potentials, limited simulation cell size and simulation time, incomplete reproduction of measured physical properties, etc.).

With the goal of better modeling radiation effects of interest to embrittlement a hybrid approach has evolved in which the nascent defect production results of $\mathrm{MD}$ simulations have been used as input to rate theory models $[1-4,11]$. MD simulations in pure $\mathrm{Fe}$, over the time scale of many of picoseconds, show that primary defect production varies with the energy of the initiating recoil atom. High-energy, neutron-induced recoil cascades induce the formation of point defect clusters (a.k.a. cascade remnants) and a reduction in the total number of defects relative to NRT predictions. In contrast, low-energy recoils result in less clustering and increased total defect survival approaching that predicted by NRT.

Experimentally this "spectral effect" (i.e. the effect of variations in primary defect production caused by variations in atomic recoil energy) has been welldocumented in experiments involving defect-mediated mass transport phenomena such as radiation-induced segregation [12], radiation-enhanced diffusion [13] and void swelling [14]. However, the impact of nascent defect production variations on embrittlement is not immediately clear. Potential effects were explored in rate-theory modeling by Stoller [4] who showed that modest variations in primary defect production parameters such as interstitial/vacancy cluster fractions and defect production efficiency could affect the magnitude of predicted embrittlement substantially.

Given the sensitivity of the modeling to input derived from MD simulations, experimental validation of the impact of the primary damage state on embrittlement is desirable. With this in mind, experiments have been undertaken to compare fast neutron and electron irradiation effects on embrittlement. Electrons produce displacement damage primarily by low energy atomic recoils, while fast neutrons produce displacements from considerably higher energy recoils. As shown in Fig. 1, defect production differences between the two types of irradiating particles can be quantified by examining their primary recoil damage spectra [15]. The median recoil damage energy, $T_{1 / 2}$, the energy above and below which half the displacement damage is produced, is nearly two orders of magnitude smaller for $\mathrm{MeV}$ electron irradiation than for fast neutron irradiation. Comparison of changes resulting from neutron irradiation, in which nascent point defect clusters can form in dense cascades, with electron irradiation, where cascade formation is minimized, can provide insight into the role that the in-cascade point defect clusters have on the mechanisms of embrittlement.

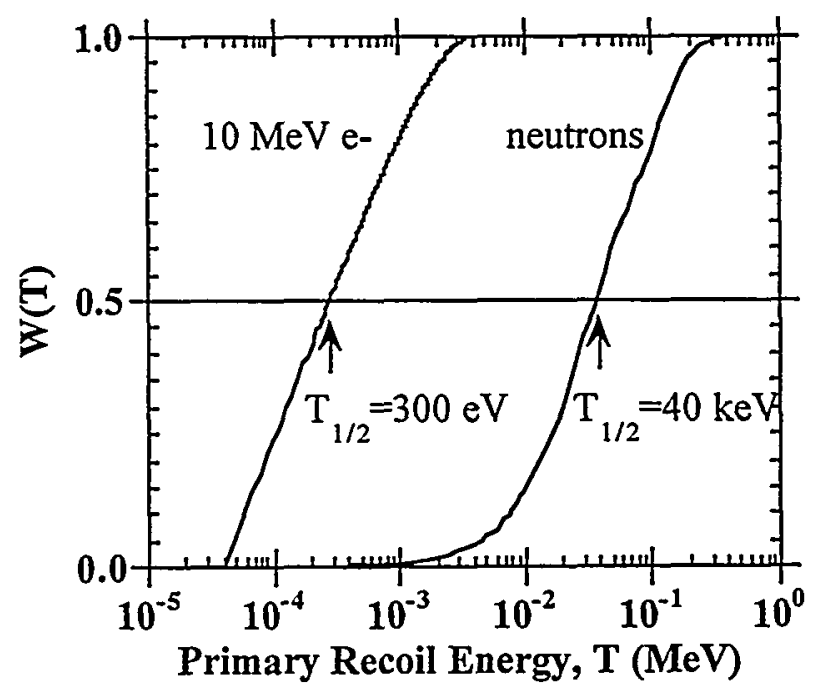

Figure 1: Integral recoil damage spectra, W(T), determined for irradiations in iron by $10-\mathrm{MeV}$ electrons and reactor neutrons. $W(T)$ is the fraction of total displacement damage produced by primary recoils with energies $\leq T$. $T_{1 / 2}$ is the median recoil damage energy for the irradiating species.

Previously, such comparative experiments performed at $60^{\circ} \mathrm{C}$ showed that primary damage state differences did not substantially alter tensile (embrittlement) behavior [16]. Comparable yield strength changes were observed in various alloys of interest to RPVs irradiated to similar displacement per atom (dpa) levels with $10-\mathrm{MeV}$ electrons or with fast neutrons. In this paper, 
mechanical property results obtained to-date for similar comparative experiments performed near the temperature of interest to commercial RPVs $\left(288^{\circ} \mathrm{C}\right)$ are examined. At this higher temperature, diffusivities are enhanced facilitating additional kinetic mechanisms (including diffusion of $\mathrm{Cu}$ ) which are consequential to embrittlement. Tensile property changes were examined in an Fe-0.9 wt.\% Cu-1.0 wt.\% $\mathrm{Mn}$ alloy and in unalloyed $\mathrm{Fe}$ subjected to $10-\mathrm{MeV}$ electron irradiation or test reactor, fast neutron irradiation.

\section{Experiments}

Miniature sheet tensile specimens with a gage section area of $9 \mathrm{~mm} \times 2 \mathrm{~mm}$ were prepared by precision die punching heat-treated wafers that had been lapped to 0.5 $\mathrm{mm}$ final thickness. The annealing treatment for both the Fe-only and Fe-0.9 wt.\% Cu-1.0 wt.\% Mn model alloy prior to wafer thinning and punching consisted of annealing at $775^{\circ} \mathrm{C}$ for 17 hours followed by a salt quench at $450^{\circ} \mathrm{C}$ for 3 minutes (no subsequent temper or stress relief). This treatment resulted in an average grain diameter of around $75 \mu \mathrm{m}$ in the ternary alloy and one slightly larger in unalloyed $\mathrm{Fe}$.

Both model alloy and $\mathrm{Fe}$ tensile samples were electron irradiated with $10-\mathrm{MeV}$ electrons from a pulsed linear accelerator operating at $60 \mathrm{~Hz}$. The slightly defocused beam entered a helium backfilled target chamber held at $0.7 \mathrm{~atm}$ and passed through a $12 \mathrm{~mm}$ square, watercooled collimator which defined the irradiation area on the gage section of the samples. To assist irradiation uniformity over the gage sections, the electron beam was wobbled slightly about $\pm 2 \mathrm{~mm}$ at rates of $4.5 \mathrm{~Hz}$ and $0.7 \mathrm{~Hz}$ in the horizontal and vertical directions, respectively. Four samples stacked two deep allowed for 8 tensile specimens to be irradiated in a single loading. Samples were heated by the electron beam and the current required to maintain the center gage section at the target temperature of $300^{\circ} \mathrm{C}$ was about $18 \mu \mathrm{A}$. For a medium $Z$ material, assuming a damage cross section of 96 barns (based on Oen's compilations [17] assuming a threshold displacement energy of $40 \mathrm{eV}$ and with correction for energy loss), this corresponded to a damage rate of $7.5 \times 10^{-9} \mathrm{dpa} / \mathrm{s}$. In order to monitor temperature, one of the specimens was outfitted with type-K thermocouples spot-welded at the center of, and just outside the top and bottom extent of the gage section. During the irradiation, a temperature gradient was present across the length of the samples with the ends of the gage section typically about $25^{\circ} \mathrm{C}$ cooler than the center held at $300^{\circ} \mathrm{C}$.

The same type of mini-tensile specimens were irradiated with fast neutrons at $290^{\circ} \mathrm{C}$ in two different experiments in the Ford Nuclear Reactor at the University of Michigan. The more recent experiment used the
Irradiation Variable Facility (IVAR) which was constructed in collaboration with University of California-Santa Barbara, Oak Ridge National Laboratory and the US Nuclear Regulatory Commission. This experiment involves an extensive test matrix including a variety of model alloys subjected to three different damage rates and irradiation temperatures. A higher fluence set of data from an older, "piggy back" experiment is also included here. In both experiments, samples were in aluminum capsules in a dry inert gas environment during the irradiation and held in the pool region immediately adjacent to the east face of the reactor core. The IVAR facility allows continuous monitoring of temperature and power during reactor cycles lasting 10 days. While $\mathrm{Fe}$ and $\mathrm{Ni}$ dosimeter wires were included in each capsule, they have not yet been analyzed and the flux and fluence values used in this work are based on a prior, preliminary dosimetric evaluation of the IVAR region. Neutron damage rates for this experiment are listed in Table I and were determined assuming a spectralaveraged damage cross section of 1500 barns.

Table I Experimental Damage Rates

\begin{tabular}{|l|c|c|}
\hline & $\begin{array}{c}\text { Damage } \\
\text { Rate } \\
(\mathrm{dpa} / \mathrm{s})\end{array}$ & $\begin{array}{c}\text { Ratio } \\
\text { relative to } \\
\text { IVAR Low } \\
\text { Neutron }\end{array}$ \\
\hline IVAR Low Neutron & $9.0 \times 10^{-11}$ & 1 \\
\hline IVAR Medium Neutron & $4.7 \times 10^{-10}$ & 5 \\
\hline Piggy-Back Neutron & $1.0 \times 10^{-9}$ & 11 \\
\hline IVAR High Neutron & $1.4 \times 10^{-9}$ & 16 \\
\hline Linac Electrons & $7.5 \times 10^{-9}$ & 83 \\
\hline
\end{tabular}

Samples were tensile tested at room temperature at a strain rate of $10^{-3} \mathrm{~s}^{-1}$. At least two specimens of each fluence and alloy were tested, with the exception of the electron irradiated Fe-only samples where only one specimen of each fluence was used. Unirradiated, control specimens, associated with the same alloy forging locations as their corresponding irradiated counterparts, were also tested.

\section{$\underline{\text { Results }}$}

Fig. 2 shows the change in yield strength (YS) as a function of calculated dpa for both the electron- and neutron-irradiated samples. Displayed tensile property error bars were derived from the standard deviations of 


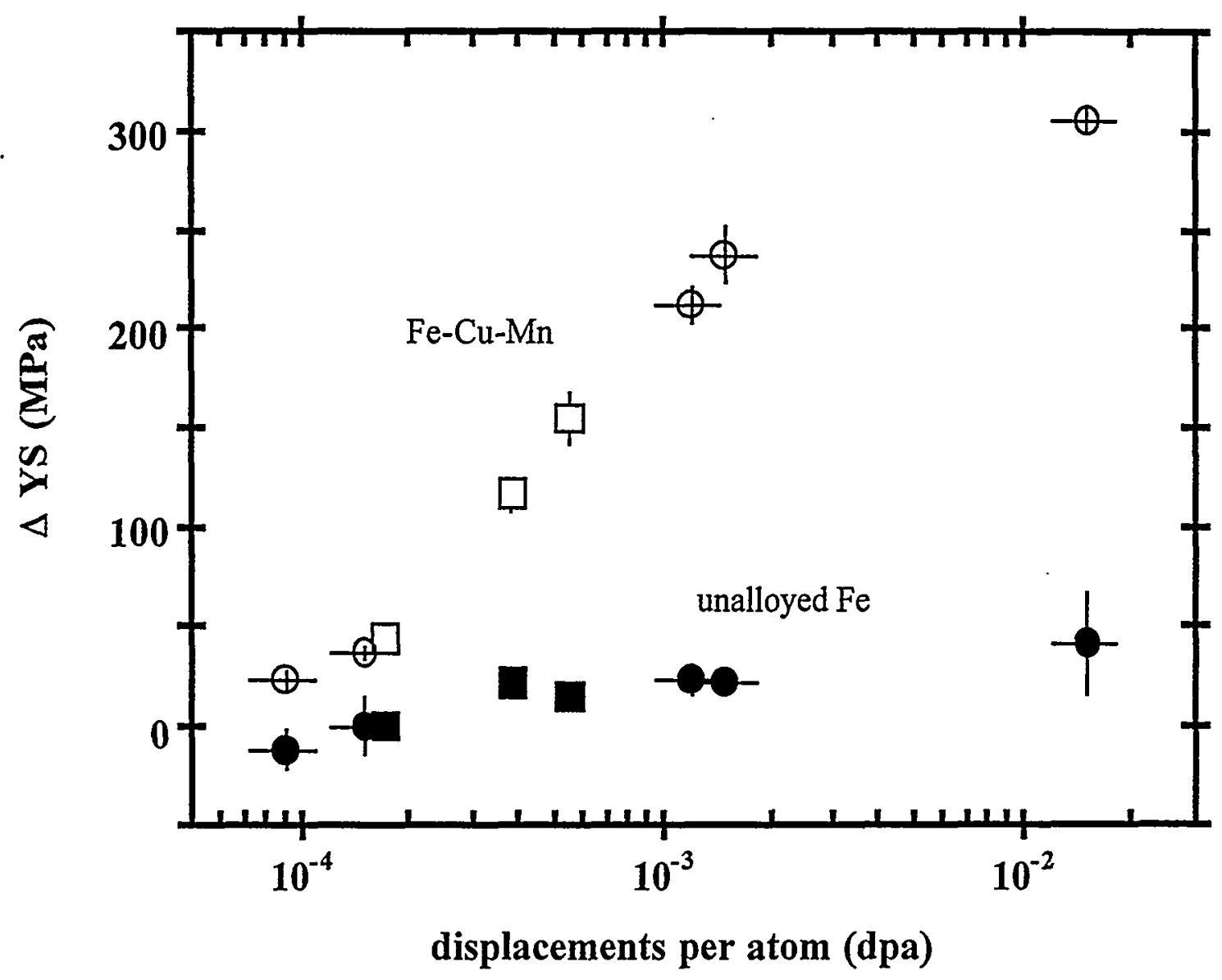

Figure 2: Change in yield strength measured at room temperature as a function of irradiation damage (dpa) at $300^{\circ} \mathrm{C}$. Squares are the $10-\mathrm{MeV}$ electron data, and circles are fast neutron data. No error bars are displayed for the electronirradiated iron data (only one sample at each damage level tested).

the respective irradiated and unirradiated measurements. Error bars of $\pm 20 \%$ were attached to the neutron data damage level data to approximately account for uncertainties in dosimetry estimates.

\section{Discussion}

The first observation readily apparent from Fig. 2 is, for the same damage level, the ternary alloy experienced substantially more embrittlement than uanlloyed $\mathrm{Fe}$ regardless of the irradiating species. The pronounced effect on embrittlement of alloying species has been well-documented in previous neutron irradiation experiments [1]. Similar behavior has also been reported in recent work examining electron irradiation-induced hardening. Barbu et al. [18] saw no increase in microhardness in pure $\mathrm{Fe}$ irradiated at $290^{\circ} \mathrm{C}$ with 2.5 $\mathrm{MeV}$ electrons to about $4.2 \times 10^{-4}$ (at a rate of $2 \times 10^{-9}$ $\mathrm{dpa} / \mathrm{s}$ ) while an $\mathrm{Fe}-0.3$ at.\% $\mathrm{Cu}$ alloy subjected to the same irradiation conditions experienced a $60 \%$ increase in hardness.
Microstructurally, the increased embrittlement in the alloy is typically attributed to the diffusive nucleation and growth of pure $\mathrm{Cu}$ or CRPs which provide an additional hardening component. Small-angle neutron scattering (SANS) experiments [1] and recent electron microscopy work are consistent with this CRP interpretation. High resolution electron microscopy (HREM) in higher Cu-content ( $\geq 1.3 \mathrm{wt} . \% \mathrm{Cu}$ ) binary alloys shows evidence for pure $\mathrm{Cu}$ precipitates forming subsequent to electron- or neutron-irradiation $[19,20]$. Reactor neutron irradiation at $270^{\circ} \mathrm{C}$ to damage levels of $8.61 \times 10^{-3} \mathrm{dpa}$ and $6.3 \times 10^{-2} \mathrm{dpa}$ resulted in mostly spherical precipitates with similar diameter ranges of 2$4 \mathrm{~nm}$. HREM work on binary alloy irradiated at $295^{\circ} \mathrm{C}$ with $2.5 \mathrm{MeV}$ electrons to $1.4 \times 10^{-3} \mathrm{dpa}$ (at a rate of $2 \times 10^{-9} \mathrm{dpa} / \mathrm{s}$ ) also show pure $\mathrm{Cu}$ precipitates with the same 9R twinned structure observed in neutron irradiated and in the annealed-only alloy. The microstructural similarity with thermally annealed material supports the idea that irradiation, whether by electrons or neutrons, enhances the precipitation process by increasing the point defect concentrations mediating 


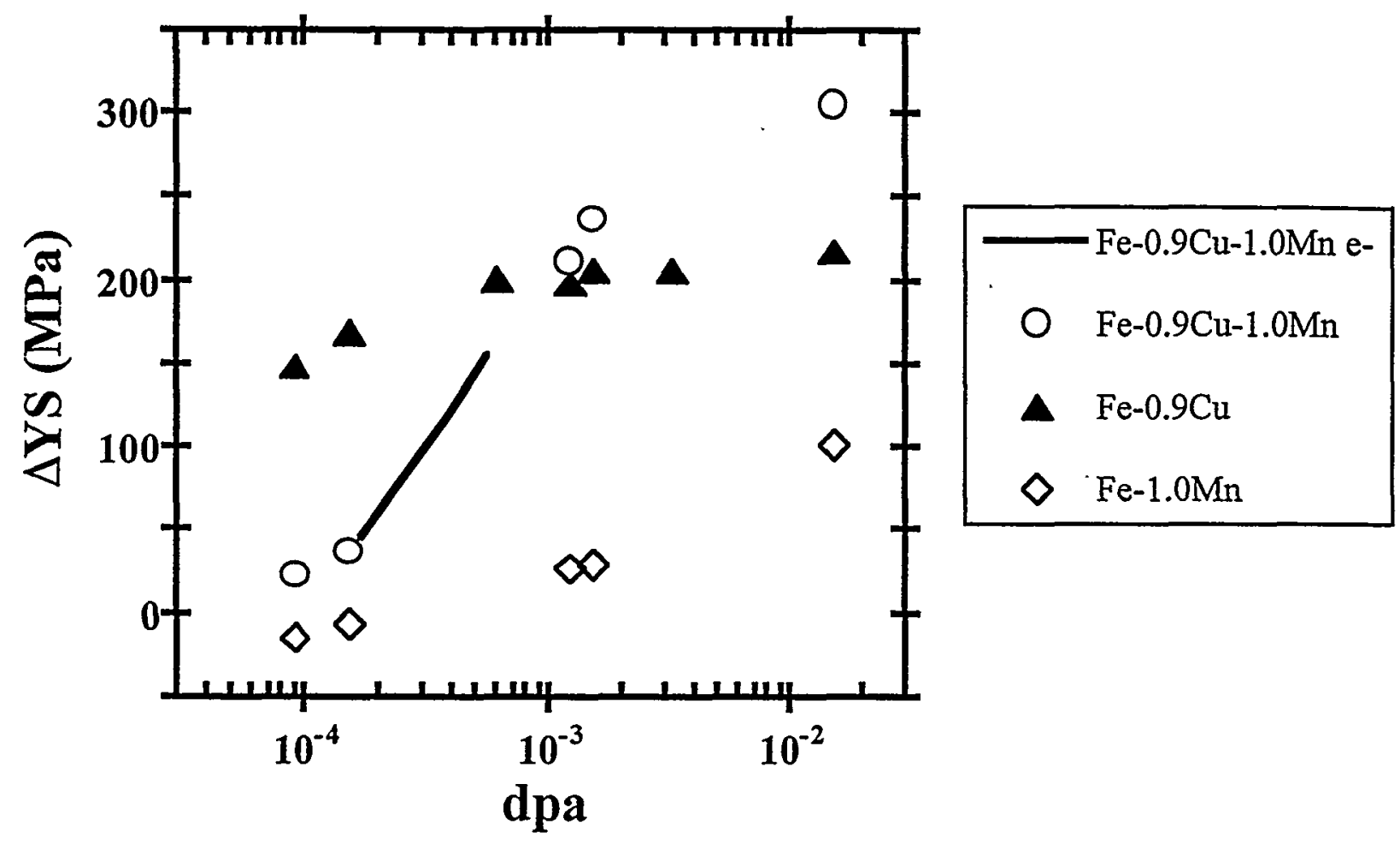

Figure 3: Change in yield strength for various model alloys neutron irradiated near $300^{\circ} \mathrm{C}$. Solid line represents existing electron data for comparison. Alloy compositions given in weight percent.

thermal diffusion. While HREM is not sensitive to the coherent BCC structure believed characteristic of small $\mathrm{Cu}$ precipitate sizes in this binary alloy, the larger diameter range of the non-coherent precipitates observed at lower damage level in the electron irradiated material $(4-17 \mathrm{~nm})$ versus that for the higher damage level neutron irradiated alloy $(2-4 \mathrm{~nm})$ is consistent with the notion that electrons are more efficient than neutrons at promoting radiation-enhanced diffusion.

A second observation from Fig. 2 is the similarity in tensile property change trends with radiation damage for electrons and neutrons. This same embrittling efficiency per dpa for both irradiating species remains qualified by the fact that different damage rates were used in the various experiments. As seen in Table I, there exists a factor of 83 difference between the highest damage rate (linac electrons) and the lowest neutron damage rate. Among only the neutron irradiations, a more modest factor of 16 difference exists between the highest and lowest damage rates.

A more sound experimental basis upon which to comment on damage rate effects will be available in the near future with additional IVAR neutron test matrix data. In the interim however, damage rate effect differences are discounted by considering model predictions and additional existing data from the neutron irradiation test matrix. Although rate theory modeling has not been applied to analyze the specific results of our experiment, the cluster-recombination rate theory model of Odette [1] suggests that there should be only a minimal to modest effect on hardening for this difference, at the damage rate levels studied. This prediction appears supported by neutron irradiated data for an Fe-Cu alloy (taken from the same IVAR and "piggy-back" experiments) as shown in Fig. 3. Despite the factor of 16 variation in damage rate, a smooth, continuous trend in yield strength change with increasing damage is observed in the $\mathrm{Fe}-\mathrm{Cu}$ alloy. At damage levels $\geq 5 \times 10^{-4} \mathrm{dpa}$, a saturation hardness level is achieved and is apparently unaffected by damage rate differences that exist among the data in this fluence range.

Assessing damage rate effects on embrittlement trends for electrons is less straight forward in lieu of experimental data or comprehensive modeling. A simple, qualitative approach would be to assume that during electron irradiation embrittlement evolves solely from diffusion of point defects which contribute to formation of defect clusters and CRPs. Steady-state rate theory would then predict that at low damage rates, fixed-sink (e.g. dislocation) dominated kinetics would ensue and the embrittlement rate (mechanical property 
change per dpa) would be constant, independent of the electron damage rate (similar to the neutron behavior described above). In the limit of high damage rates, mutual recombination of point defects dominates the kinetics and the embrittlement rate would decrease with increasing damage rate according to (damage rate) ${ }^{-1 / 2}$.

With these interpretations of damage rate effects, we can identify two possible qualitative conclusions regarding the embrittling efficiencies of the electrons and neutron irradiations used in this work. If the electron irradiation damage rate is considered to be in the damage rate independent regime, the experimental data simply implies that electrons and neutrons are equally efficient at producing embrittlement. If however, we consider the electron damage rate to be in the damage rate dependent regime, it becomes necessary to "correct" for embrittlement rate changes due to damage rate prior to comparing the two types of irradiating species. Taking into account an anticipated embrittlement rate decrease due to high damage rate, the fact that the electron-induced tensile changes follow the same trend as those induced by the lower damage rate neutrons (Fig. 2) suggests that the electrons are more efficient (on a per dpa basis) than the neutrons at causing embrittlement.

Some insight into the mechanism affecting embrittlement in the low damage regime $\left(<10^{-3} \mathrm{dpa}\right)$ where the neutron and electron effects appear similar may be gained by further examining Fig. 3 . If the embrittlement trend observed in the neutron irradiated Fe-Mn alloy is solely attributed to matrix damage, it's trend with dpa damage observed in Fig. 3 suggests that defect clusters (produced for example within neutroninduced cascades) contribute minimally to yield strength changes in the ternary alloy within the range where the greatest increase in embrittlement is observed (i.e. between $10^{-4}$ and $10^{-3} \mathrm{dpa}$ ). This implies that CRP formation is the dominant hardening source in this dpa range. Furthermore, it is apparent when compared with the Fe-Cu alloy in Fig. 3, the presence of $\mathrm{Mn}$ acts to delay precipitation in the ternary alloy whether irradiated with electrons or neutrons.

Clearly, further understanding the surprising similarity in embrittlement behavior between electrons and neutrons awaits detailed microstructural characterization of the irradiation embrittled materials. The results for this experiment indicate that the substantial primary damage state differences known to exist between electrons and neutrons do not always exert a corresponding influence on the aggregate changes in mechanical properties. This may suggest that future modeling efforts must carefully consider the balance of competing effects that may be produced by various types of primary sources of displacement damage on all the multiscale-multiphysics processes [21] leading to hardening and embrittlement.

\section{Conclusions}

Changes in yield strength and ultimate tensile strength resulting from $10 \mathrm{MeV}$ electron- or test reactor neutronirradiation at $300^{\circ} \mathrm{C}$ revealed a strong effect of small alloying additions to iron. Regardless of the irradiating species, an $\mathrm{Fe}-0.9$ wt.\% $\mathrm{Cu}-1.0$ wt.\% $\mathrm{Mn}$ alloy experienced substantially greater tensile changes compared to unalloyed $\mathrm{Fe}$ in the damage range of $9.0 \times 10^{-5} \mathrm{dpa}$ to $1.5 \times 10^{-2} \mathrm{dpa}$. This result is consistent with previous work demonstrating the addition of a Curelated precipitation component of hardening in the alloy.

Similar trends in tensile property changes with increasing radiation damage were observed for the electrons and neutrons in this work. Considering damage rate effects, this result could be interpreted as the electrons being equal to, or more efficient than, fast neutrons at causing embrittlement depending on the damage rate regime assumed for the electron irradiations.

\section{Acknowledgements}

This work supported by the U.S. Department of Energy, BES-Materials Sciences, under contract \#W-31-109ENG-38.

\section{References}

1. G.R. Odette, and G.E. Lucas, Radiation Effects and Defects in Solids, 144 (1998), 189-231.

2. G.R. Odette, in Microstructure of Irradiated Materials, Vol. 373, ed I. M. Robertson, L. E. Rehn, S. J. Zinkle and W. J. Phythian (Materials Research Society, Pittsburgh, PA, 1995), 137-148.

3. E.V. Mader., "Kinetics of Irradiation Embrittlement and the Post-Irradiation Annealing of Nuclear Reactor Pressure Vessel Steels" (Ph.D. Thesis, University of California, Santa Barabara, 1995).

4. R.E. Stoller, "A Comparison of the Relative Importance of Copper Precipitates and Point Defect Clusters in Reactor Pressure Vessel Embrittlement", Oak Ridge National Laboratory NUREG/CR-6231, 1994.

5. Stoller, R. E., G.R. Odette and B.D. Wirth, Journal of Nuclear Materials 251 (1997), 49-60.

6. W.J. Phythian, R.E. Stoller, A.J.E. Foreman, A.F. Calder and D.J. Bacon, Journal of Nuclear Materials 
223 (1995), 245-261.

7. N. Soneda and T. Diaz de la Rubia, Philosophical Magazine A 78 (1998), 995-1019.

8. F. Gao, D.J. Bacon, P.E.J. Flewitt and T.A. Lewis, in Microstructure Evolution During Irradiation, Vol. 439, ed I.A. Robertson, G.S. Was, L.W. Hobbs and T.D. de la Rubia (Materials Research Society, Pittsburgh, PA, 1997), 307-318.

9. A.F. Calder and D.J. Bacon, ibid, 521-526.

10. D.J. Bacon, A.F. Calder and F. Gao, Journal of Nuclear Materials 251 (1997), 1-12.

11. Y. Katoh and T. Muroga, Journal of Nuclear Materials 233-237 (1996), 1029-1034.

12. L.E. Rehn and H. Wiedersich, Materials Science Forum 97-99 (1992), 43-58.

13. H. Wollenberger, V. Naundorf, and M.-P. Macht, "Radiation-Induced Diffusion in Nuclear Materials" in Diffusion Processes in Nuclear Materials, ed R.P. Agarwala (Elsevier Science Publishers B.V., Amsterdam, The Netherlands, 1992).

14. L.K. Mansur and P.R. Okamoto, Nuclear Metallurgy 18 (1973), 509.

15. R.S. Averback, Journal of Nuclear Materials 216 (1994), 49-62.

16. D.E. Alexander, K. Farrell and R.E. Stoller, Journal of Nuclear Materials 228 (1996), 68-76.

17. O.S. Oen, "Cross Sections for Atomic Displacements in Solids by Fast Electrons", Oak Ridge National Laboratory ORNL-4897, 1973.

18. A. Barbu,, M.H. Mathon, F. Maury, J.F. Belliard, B. Beuneu and C.H. de Novion, Journal of Nuclear Materials 257 (1998), 206-211.

19. H.A. Hardouin Duparc, R.C. Doole, M.L. Jenkins and A. Barbu, Philosophical Magazine Letters 71 (1995), 325-333.

20. A.C. Nicol, M.L. Jenkins and M.A. Kirk, in Microstructural Processes in Irradiated Materials, Vol. 540, ed S.J. Zinkle, G.E. Lucas, R.C. Ewing and J.S. Williams (Materials Research Society, to be published 1999), 409-414.

21. These processes, which range from primary defect production, through nanostructural evolution to structure property relations, are discussed in Reference [1]. 